\title{
SCHMIDT NUMBER OF SAND SUSPENSIONS UNDER OSCILLATING GRID TURBULENCE
}

\author{
Daniel Buscombe ${ }^{1}$ and Daniel C. Conley ${ }^{2}$
}

\begin{abstract}
In many models of sand suspension under waves, the diffusivity of sediment is related to the diffusivity of momentum by the inverse of the turbulent Schmidt number. The value and parameterization of this number has been the topic of much research, yet a lack of consensus has led to ad hoc adjustments in models of turbulent sediment suspensions, with apparently little physical justification. In order to study sediment diffusivity we conducted laboratory experiments to generate gradient-only sediment diffusion. Concentrations of sand suspended by near-isotropic turbulence generated by an oscillating grid, together with detailed velocity measurements, were used to calculate vertical profiles of the Schmidt number with a range of grain sizes and flow conditions. Initial results suggest that momentum diffusivity is greater than sediment diffusivity, and that the ratio of the two scales with grid Reynolds number. Ongoing work will ascertain whether an apparent grain size dependence could instead be explained by two-way feedbacks between sediment and turbulence.
\end{abstract}

Keywords: oscillating grid turbulence; Schmidt number; sediment diffusivity; suspended sediment; eddy viscosity

\section{INTRODUCTION}

In nearshore (combined wave and current) flows, fluid velocities and sand concentrations vary strongly in time during a wave cycle (Conley and Beach, 2003) which is why so-called (wave) 'phaseresolving' or 'intra-wave' models of suspended sediment transport have gained in popularity in recent years (e.g. Li and Davies, 2001; Holmedal et al., 2004; Henderson et al., 2004; Conley et al., 2008; Ruessink et al., 2009). This type of model predicts the velocity and sand concentration fields in time and space by combining solutions to the basic fluid momentum and continuity equations with an advection-diffusion equation to compute the sediment mass balance. form:

Suspended sand concentrations are obtained by solving a 1DV advection-diffusion equation of the

$$
\frac{\partial C}{\partial t}=\frac{\partial}{\partial z}\left(\varepsilon_{s}(z) \frac{\partial C}{\partial z}+w_{s} C\right)
$$

where $C=$ instantaneous volumetric sand concentration; $t=$ time; $z=$ vertical coordinate; $\varepsilon_{\mathrm{s}}=$ sediment diffusivity; and $w_{\mathrm{s}}=$ sand settling velocity. Describing fluid motion requires a corresponding momentum balance equation (e.g. Li and Davies, 2001), the solution of which requires an expression for turbulent eddy viscosity which describes the fluid turbulence. In the approach to modelling turbulent mixing under nearshore waves described above, the simplest treatment of sediment diffusivity is to express it as some fraction of the turbulent momentum diffusivity. The ratio, known as the Schmidt number, in nearshore sediment transport models is the ratio of the turbulent eddy viscosity, $v_{t}$, to sediment diffusivity, $\varepsilon_{\mathrm{s}}$ :

$$
\beta=\frac{v_{t}}{\varepsilon_{s}}
$$

Outputs of phase-resolving models of sediment suspension are very sensitive to the Schmidt number (e.g. Davies, 1995; Amoudry et al., 2005; Ruessink et al., 2009). Many models assume $\beta=1$ (e.g. Fredsoe et al., 1985, Celik and Rodi, 1988), an assumption which seems safest when the evidence for its value seems so contradictory. Indeed there are approximately as many studies in the literature which have used a value less than 1 as those which have a value greater than 1 . An argument commonly stated for $\beta>1$ is that particles lose correlation with fluid motion as they settle through turbulent eddies (e.g. Fredsoe and Diegaard, 1992). A counter argument (which leads to $\beta$ $<1$ ) is that centrifugal forces have a larger effect on particles than they do on the surrounding fluid, due to particle inertia, thought to be the case above a rippled bed (e.g. van Rijn, 1984; Davies and Thorne, 2005).

Nearshore sediment transport literature reports values between 0.1 and 10 . This large variation inadequate parameterization of $\beta$, which is therefore allowed to vary with model equations and boundary conditions used. The Schmidt number is often used as a tunable parameter (e.g. Ruessink et al., 2009), which isn't a satisfactory situation.

1 School of Marine Science and Engineering, Plymouth University, Drake Circus, Plymouth, Devon, PL4 8AA, UK

2 School of Marine Science and Engineering, Plymouth University, Drake Circus, Plymouth, Devon, PL4 8AA, UK 
This study is motivated by the observation that it should be possible to calculate Schmidt numbers stationary sediment suspension created by gradient diffusion. Such a situation is realized under homogeneous and isotropic turbulence fields with zero-mean shear created by oscillating grids.

Time-averaged concentrations of suspended sediment in zero-mean flows arise from a balance between an upward mixing flux and a downward settling flux. In classical gradient (Fickian) diffusion the upward mixing flux is proportional to the concentration gradient. Therefore the time-averaged mass balance may be represented by:

$$
\varepsilon_{s} \frac{d \bar{C}}{d z}-\bar{C}(z) w_{s}=0
$$

where $z$ is the vertical coordinate increasing upwards from the centre of grid shaking, and the overbar denotes time-average, therefore sediment diffusivity may be written:

$$
\varepsilon_{s}=\frac{-w_{s} \bar{C}}{\frac{d \bar{C}}{d z}}
$$

The statistical characteristics of oscillating grid turbulence are well known (e.g. Hopfinger and Toly, 1976; Matsunaga et al., 1999). As well as a number of studies investgating only the properties of turbulence, oscillating grids have also been used to study the iniation of non-cohesive sediment motion (e.g. Medina et al., 2001), and more commonly used for cohesive sediment dynamics, for example sediment-induced stable stratification, lutocline development and turbulence-damping (e.g. Michallet and Mory, 2004; Gratiot et al., 2005). This study is the first to the authors' knowledge to use oscillating grid turbulence to look specifically at non-cohesive sediment diffusivity.

\section{OSCILLATING GRID EXPERIMENTS AND MEASUREMENTS}

We constructed a turbulence tank with dimensions $50 \mathrm{~cm}$ long by $50 \mathrm{~cm}$ wide by $80 \mathrm{~cm}$ deep (Figure 1). The grid consisted of $7 \times 71 \mathrm{~cm}$ bars and $5 \mathrm{~cm}$ square holes. This ratio of hole to bar width is a standard design criteria because, with a grid porosity of $65 \%$, it has been shown by Hopfinger and Toly (1976) to be the most efficient at reducing secondary flows within the tank.

\section{Linear actuator}

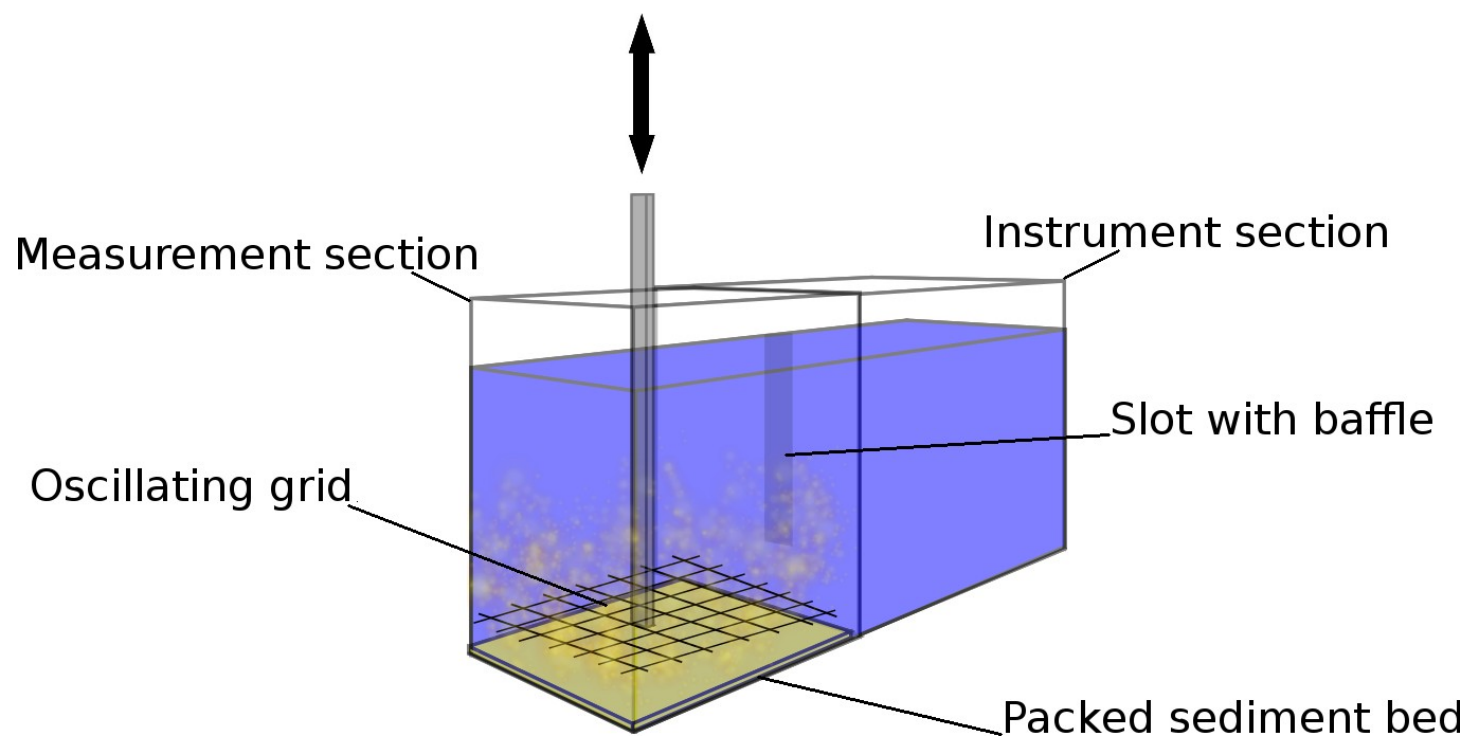

Figure 1. Schematic of the oscillating-grid turbulence tank used in this study. There are two sections to the tank separated by a wall with a baffled slot. This is in order for probe heads to be inserted through the wall with minimal flow disturbance.

The tank was filled with fresh tap water at $20^{\circ} \mathrm{C}$. A programmable magnetic linear actuator has been used instead of the more traditional armed cam or 'scotch crank' type grid oscillating motor. The linear actuator permits greater stroke lengths than traditional motors, and also a wider range of waveforms (skewed or asymmetrical, even irregular waveforms). Larger stroke lengths are advantageous because the grid Reynolds number goes with the square of the oscillation stroke, $S$ : 


$$
\operatorname{Re}_{\text {grid }}=\frac{f S^{2}}{v}
$$

where $v$ is kinematic viscosity and $f$ is oscillation frequency $(\mathrm{Hz})$. This paper presents initial results from two flow conditions: 1) a $2 \mathrm{~Hz}, 10 \mathrm{~cm}$ stroke sinusoid (Regrid $=14750$ ); and 2 ) a $3 \mathrm{~Hz}, 7 \mathrm{~cm}$ stroke sinusoid (Regrid $=10808)$. Turbulence measurements were made using a Vectrino II acoustic Doppler Profiling Velocimeter (made by AS Nortek, Norway). The Vectrino II measures a vertical profile of three components of velocity $(u, v, w)$ at high sample rates (we sampled at $100 \mathrm{~Hz}$ ). We profiled over $30 \mathrm{~mm}$ at $1 \mathrm{~mm}$ bin spacing.

Experiments were conducted using a packed sediment bed of solid glass spheres (Figure 1). This material was chosen because it has the same density as quartz sand, but not the variation in particle shape. Therefore, reduced uncertainty in the application of both formulae for settling velocity from measured particle size, and concentration estimates using an acoustic backscatter sensor (ABS). For a review of ABS technology see Thorne and Hanes (2002). This paper presents results using glass spheres with narrow and non-overlapping size ranges: 1) 70-100 micron (hereafter 'fine'); and 2) 145205 micron (hereafter 'coarse'). The scattering acoustic properties of spheres, which was used to calculate concentration from ABS returns, are well documented and an exact solution exits (Gaunaurd and Uberall, 1983).

Sediment concentrations were measured using a 1, 2 and $4 \mathrm{MHz}$ ABS (AQUAscat 1000 made by Aquatec, UK). The three transducer-receivers were deployed downward-looking, profiling over $60 \mathrm{~cm}$ with $5 \mathrm{~mm}$ bins, from just below the free surface to the region of grid shaking. In addition, physical samples were taken during experiments using a pump-sampler consisting of five intake tubes $(5 \mathrm{~mm}$ internal diameter), pumping water and sediment at a rate of $2 \mathrm{~L} / \mathrm{min}$. This pump sampler is operated and profiled vertically whilst the grid is shaking. Physical samples were vacuum-filtered, dried and weighed for mass concentration estimates. Grain-size distributions were obtained from replicates of each sample using a laser-diffraction instrument in the laboratory.

\section{DATA PROCESSING}

The size of the fine sediment was selected so it has a grain Reynolds number of $<1$. The particle Reynolds number is given by:

$$
\operatorname{Re}_{\text {grain }}=\frac{w_{s} D}{v}
$$

where $D$ is grain diameter and $w_{s}$ is settling velocity. When $\operatorname{Re}_{\text {grain }}<1$, Stokes' Law of settling applies, in which settling velocity is given by:

$$
w_{s}=\frac{R g D^{2}}{c_{1} v}
$$

where $R=1.65$ is the submerged specific gravity of quartz in water, and $\mathrm{c}_{1}$ is a coefficient (exactly 18 for smooth spheres). The coarse sediments have $R e_{\text {grain }}>1$, so the turbulent effects of the particle settling through the still water must be taken into account using the formula of Ferguson and Church (2004), where $c_{2}$ is a coefficient (exactly 0.4 for smooth spheres):

$$
w_{s}=\frac{R g D^{2}}{c_{1} v \sqrt{0.75 \mathrm{C}_{2} \operatorname{Rg} D^{3}}}
$$

Measured grain-size distributions showed very little variation with depth. Therefore only the depth-averaged grain diameter was used which for the fine sediment was $100 \mu \mathrm{m}$, and $170 \mu \mathrm{m}$ for the coarse sediment. The computed settling velocities using (7) and (8) for fine and coarse sediment were $0.0093 \mathrm{~ms}^{-1}$ and $0.0136 \mathrm{~ms}^{-1}$, respectively.

Velocity data underwent a conservative quality control procedure where only velocities with correlations greater than $90 \%$ and amplitudes greater than $-50 \mathrm{~dB}$ were kept. Mean flows (Figure 2) were less than $2 \mathrm{~cm} \mathrm{~s}^{-1}$ for all components of velocity and grid shaking conditions. The flow was nearisotropic according to a computed index of turbulence isotropy given by (Redondo et al., 2001):

$$
I=\frac{\rho \overline{u^{\prime} w^{\prime}}}{\rho \sigma_{u} \sigma_{w}}
$$


where primes indicate the fluctuating component of the three components of velocity vectors, and $\sigma$ indicates r.m.s. Using this metric, $I=1$ when the flow is homogeneous and isotropic. All of our measurements fall in the range 0.8 to 1 , which is comparable to previous oscillating grid studies (Redondo et al., 2001).
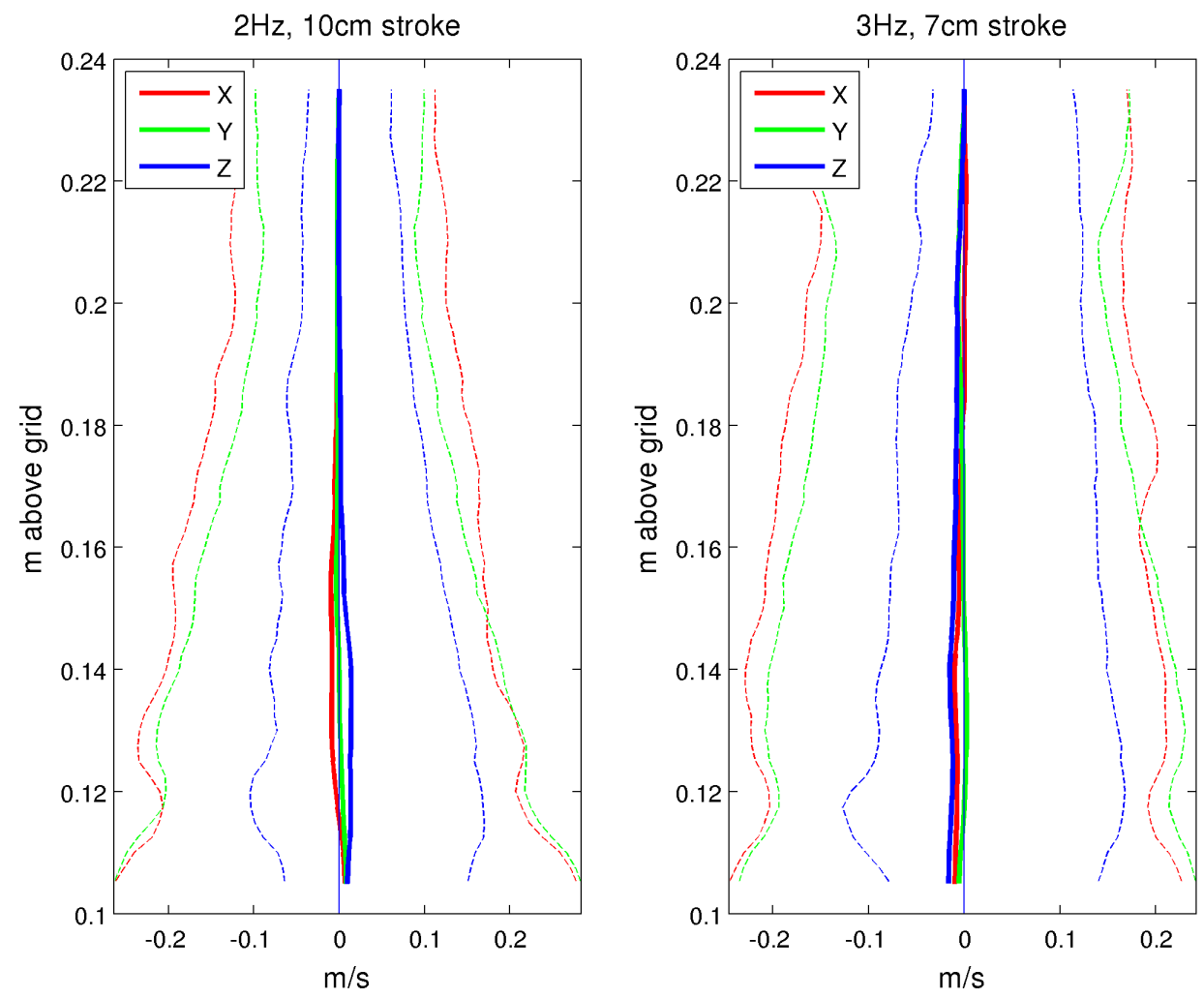

Figure 2. Vertical profiles of mean (solid lines) and maximum (dashed lines) velocities for both grid shaking conditions. Mean flows are within $2 \mathrm{~cm} \mathrm{~s}^{-1}$

Turbulent kinetic energy was computed using

$$
K=\frac{1}{2}\left(u^{\prime 2}+v^{\prime 2}+w^{\prime 2}\right)
$$

Sediment mass-concentration, $M(\mathrm{~g} / \mathrm{L})$, was obtained following the implicit iterative approach described by Thorne and Hanes (2002) where:

$$
M_{i}=\left(\frac{V_{r m s_{i}}}{k_{s_{i}} k_{t_{i}}}\right)^{2} \psi_{i}^{2} r^{2} e^{4 \mathrm{r} \alpha_{i}}
$$

where $i$ refer to the three acoustic frequencies; $V_{r m s}$ is the root mean square of the recorded instrument voltage; $\psi$ is an acoustic spreading function; $r$ is the range to the transducer; $\alpha$ is an attenuation coefficient due to water and sediment. Water attenuation is a straightforward function of water temperature, depth and salinity. Sediment attenuation is a function of $r, M$, and a total scattering cross-section. Scattering is primarily a function of particle shape, and for a suspension of spheres is well known which helps with the accuracy of the results obtained here. Coefficient $k_{t}$ is a constant which depends on the instrument electronics and is provided by the instrument manufacturer. Coefficient $k_{s}$ is a function of the attenuation of sound due to sediment, sediment density, and sediment form function which describes the backscattering characteristics of the sediment suspension. Coefficient $k_{s}$ has been obtained using the methods outlined in Betteridge et al. (2008) using an analytical form function for spheres. 
$2 \mathrm{~Hz}, 10 \mathrm{~cm}$ stroke

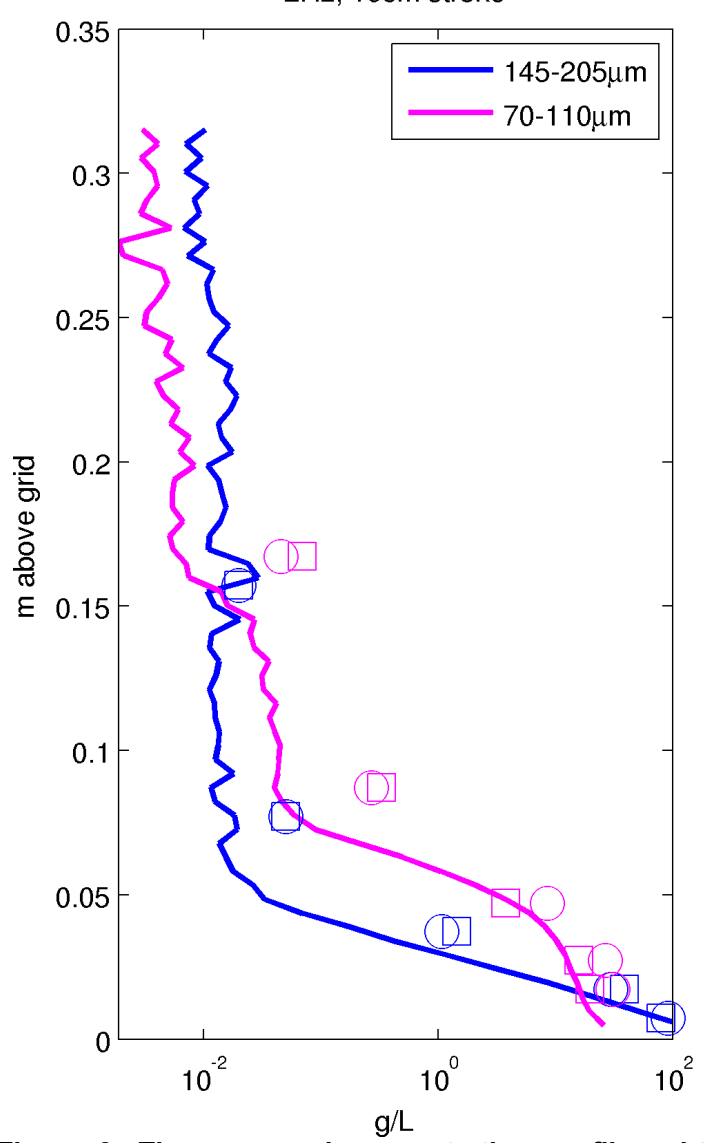

$3 \mathrm{~Hz}, 7 \mathrm{~cm}$ stroke

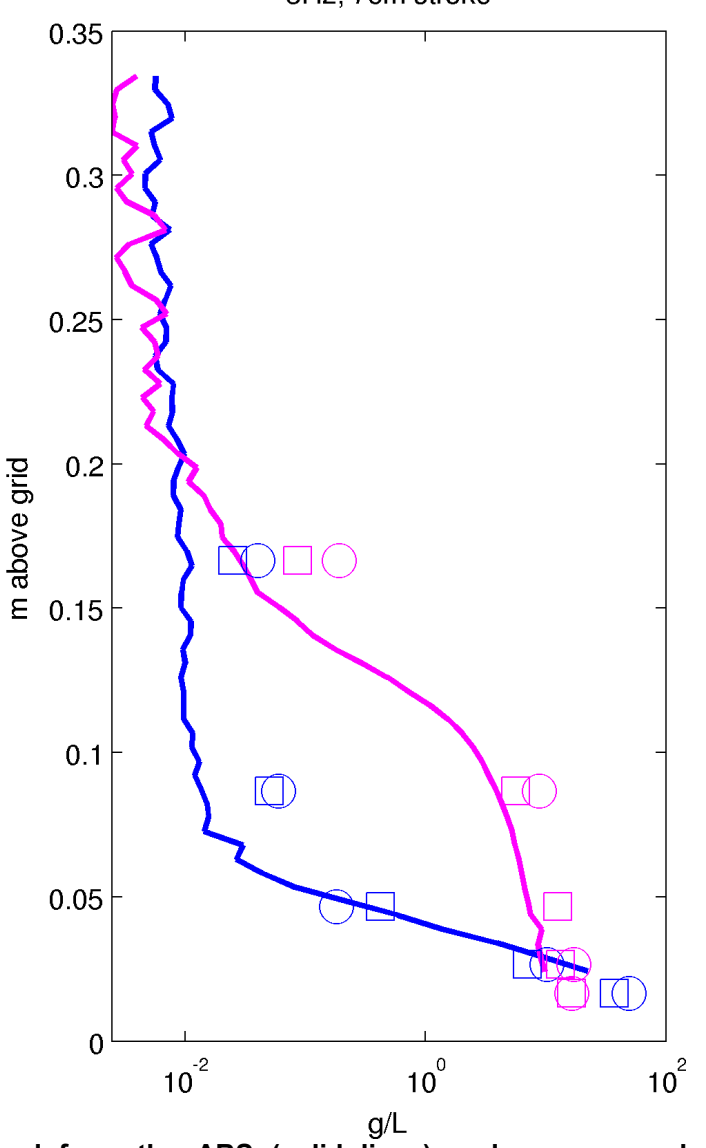

Figure 3. Time-averaged concentration profiles obtained from the ABS (solid lines) and pump sample (symbols) for both flow conditions and grain sizes

\section{RESULTS}

Time-averaged concentration profiles, obtained with the ABS, for both sets of flow (grid shaking) and both grain sizes (Figure 3) show that more sediment is suspended for the finer grain size than for the coarser. Comparisons of time-averaged concentration from the ABS and from pump samples (symbols in Figure 3) show good agreement near the grid in the high-concentration region and worse agreement with distance away from the grid. It is in this region where our confidence with the ABS concentrations is low because of the persistence of a finite and fluctuating concentration at distance from the grid, symptomatic of random noise. It is possible the ABS is measuring the hollow spheres in the water which is the seeding material required for the acoustic velocimeter.

Sediment diffusivities estimated using a simple differencing scheme given by Thorne et al., (2009):

$$
\varepsilon_{s}=\frac{\frac{-w_{s_{j}}+w_{s_{k}}}{2} \frac{\overline{C_{j}}+\overline{C_{k}}}{2}}{\frac{2}{\bar{C}_{k}}-\overline{C_{j}}} \frac{\delta_{j k}}{}
$$

are shown as vertical profiles in Figure 4. Here, $j$ and $k$ denote adjacent bins, and $\delta j k$ is $5 \mathrm{~mm}$. There is a lot of scatter due to irregularities in computed concentration gradients (the denominator in (12)), in turn due to the irregular behaviour in the time-averaged concentrations described above.

\section{ANALYTICAL EXPRESSION FOR SCHMIDT NUMBER}

Given the low confidence in concentrations far from the grid, time-averaged concentration is instead represented by a simple power-law form, where $h$ is water depth: 


$$
\bar{C}=\xi((h-z) / z)^{\gamma}
$$

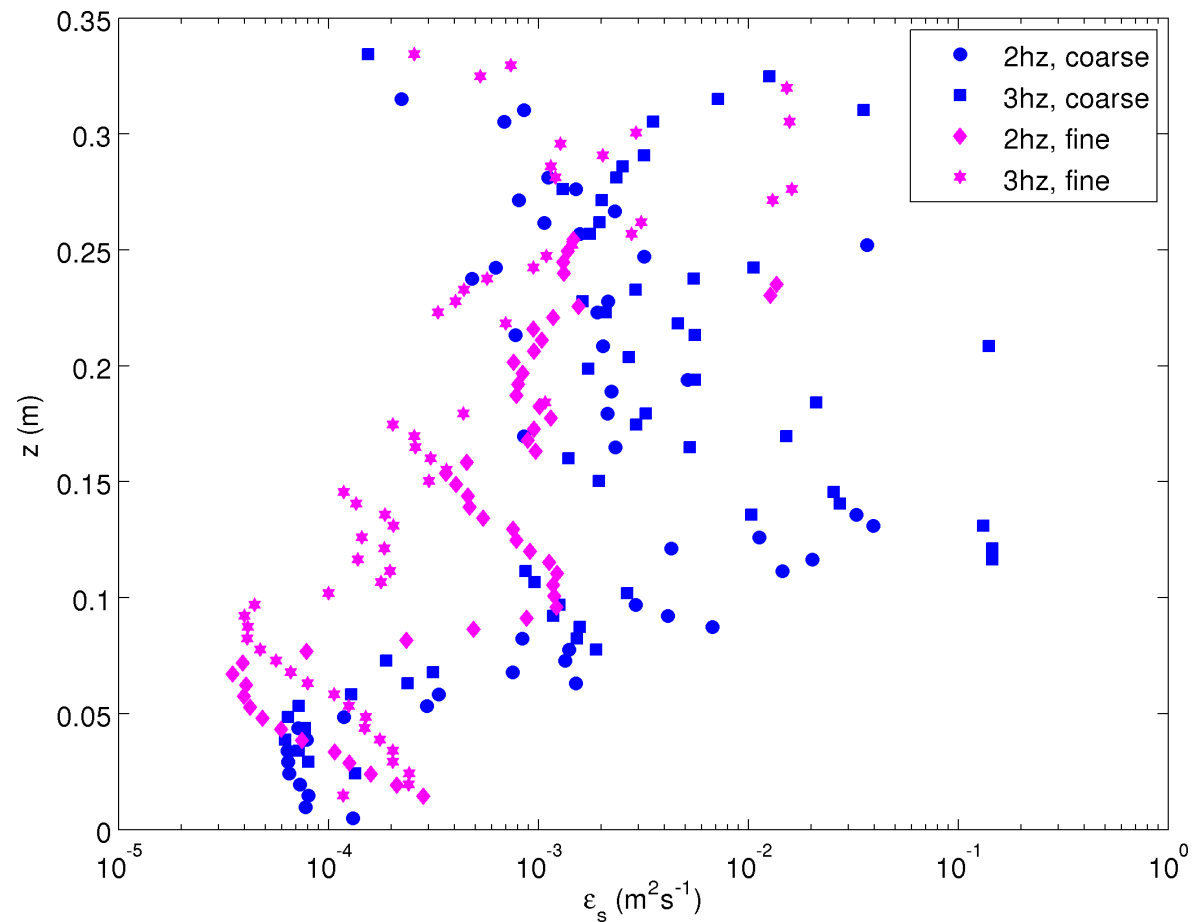

Figure 4. Sediment diffusivities callculated from the ABS concentration profiles, using (12).
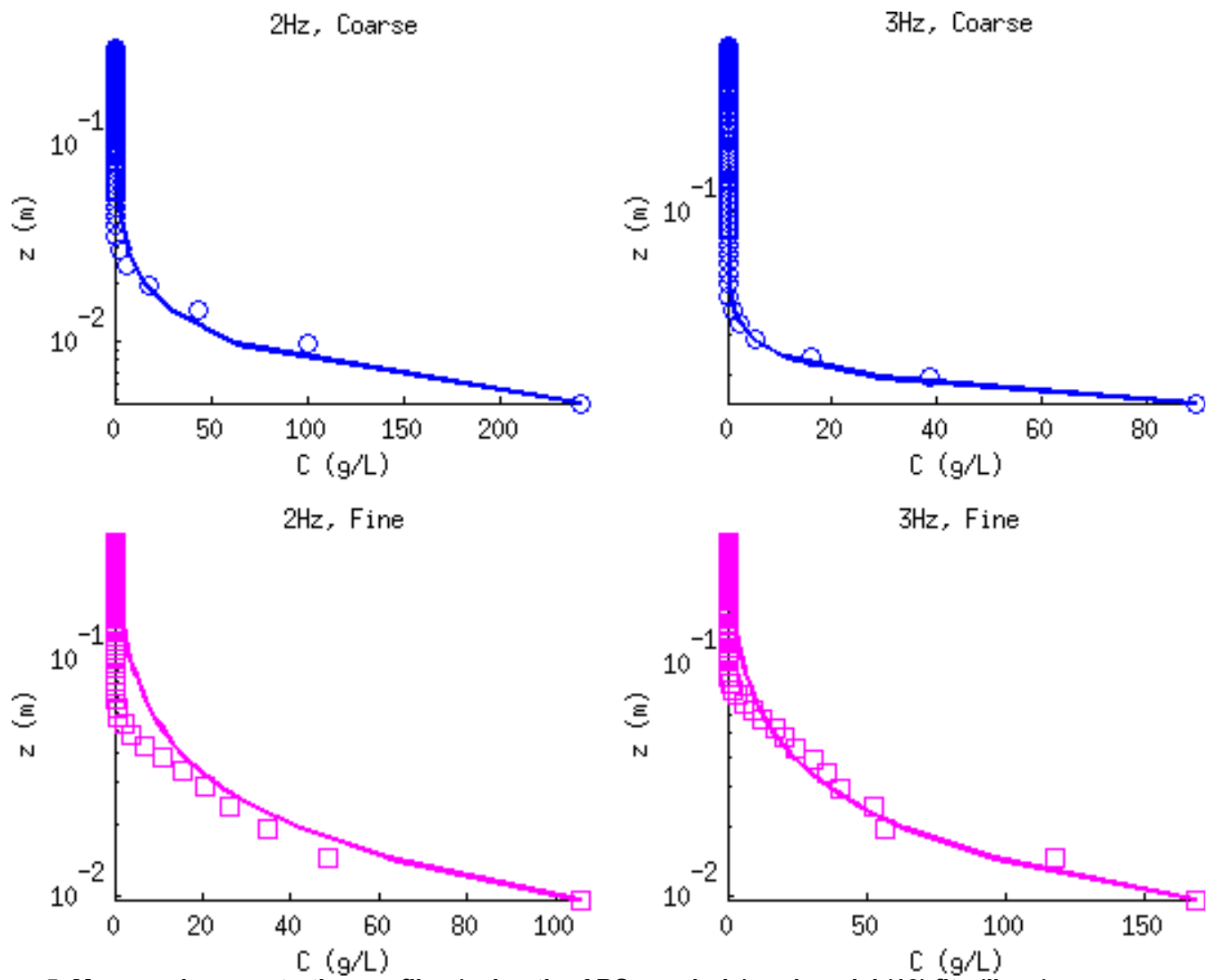

Figure 5. Measured concentration profiles (using the ABS, symbols) and model (13) fits (lines).

Coefficient $\xi$ is akin to a reference concentration at some small $z$, whereas $\gamma$ acts as a coefficient of diffusion. Therefore (13) is similar in form to a classic Rouse shape but without the specification of diffusion coefficient (Rouse parameter) in physical terms. We use least-squares methods to solve for $\xi$ 
and $\gamma$. The model fits well to the observations (Figure 5) especially where concentrations are high. Inserting (13) into (3) we obtain an analytical expression for sediment diffusivity:

$$
\varepsilon_{s}=\frac{-w_{s} \bar{C}}{\frac{-\xi \gamma h}{z^{2}}\left(\frac{h-z}{z}\right)^{\gamma-1}}
$$

The model (Figure 6) predicts a parabolic shape which is also apparent in the diffusivities calculated using (12). There is good agreement at high concentrations (small $z$ ) and worse agreement where the discrepancy between measured and modelled $C$ is large at large $z$ (when measured $C$ don't rapidly converge on zero).

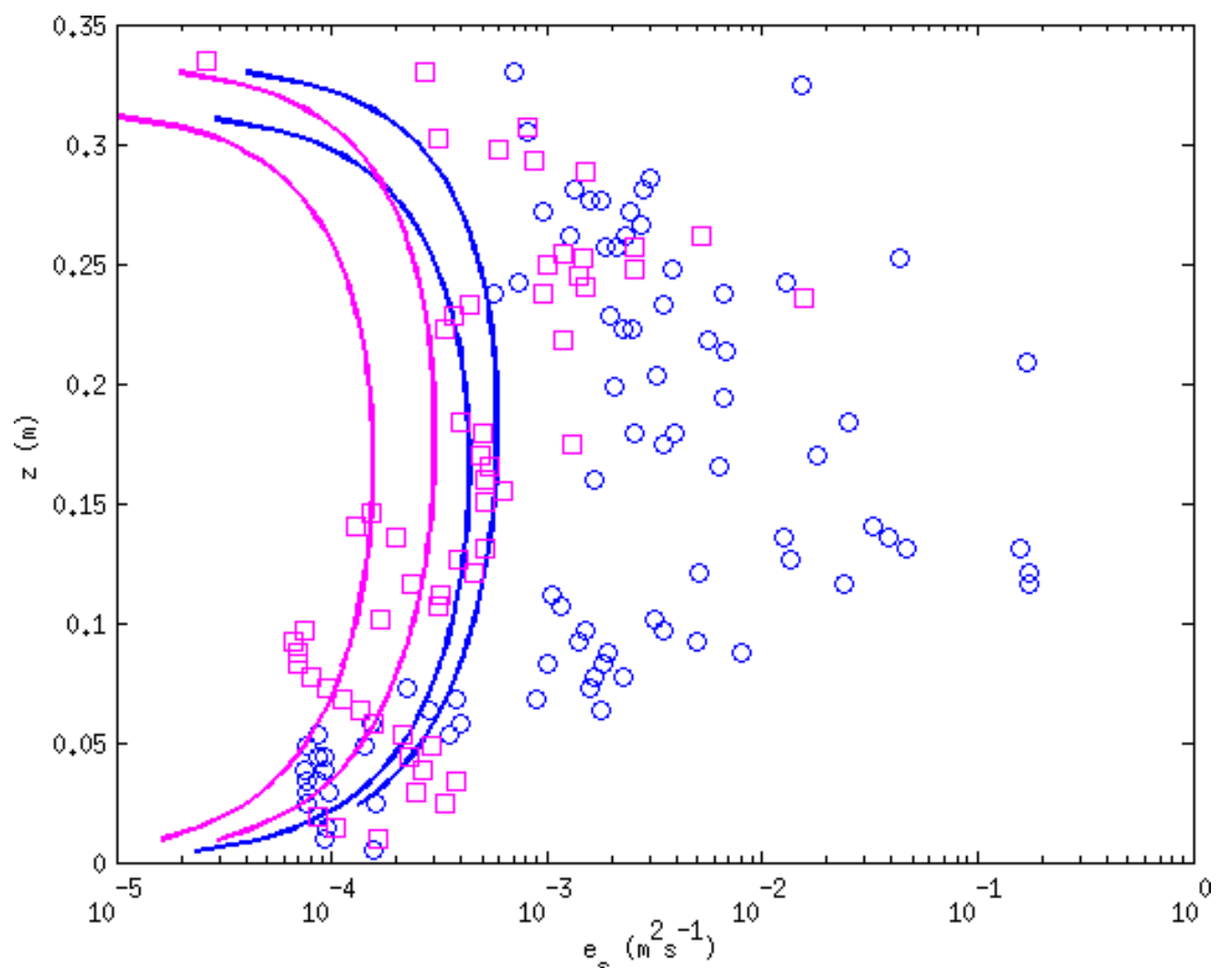

Figure 6. Measured (symbols, replicated from Figure 5) and modelled (lines, using (13)) sediment diffusivities.

In order to obtain a similar analytical expression for momentum diffusivity, we adopt the expression for $K$ given by Orlins and Gulliver (2003):

$$
K=\frac{1}{2} \omega\left(\sqrt{M} S^{1.5} f z^{-1}\right)^{2}
$$

where:

$$
\omega=2 C_{1}^{2}+C_{2}^{2}
$$

The optimal values for coefficients $C_{1}$ and $C_{2}$ are unknown but may be dependent on grid geometry. Following Orlins and Gulliver (2003), we adopted the values $C_{1}=0.22$ and $C_{2}=0.26$ as suggested by deSilva and Redondo (1992). The model for $K$ gives a reasonable agreement with our calculated $K$ using (10), especially for the $2 \mathrm{~Hz}, 10 \mathrm{~cm}$ grid shaking (Figure 7 ). Better agreement would be obtained for the $3 \mathrm{~Hz}$ shaking if different values for $C_{1}$ and $C_{2}$ were used, however it is unclear whether these coefficients should be functions of shaking frequency so this has not been attempted. 

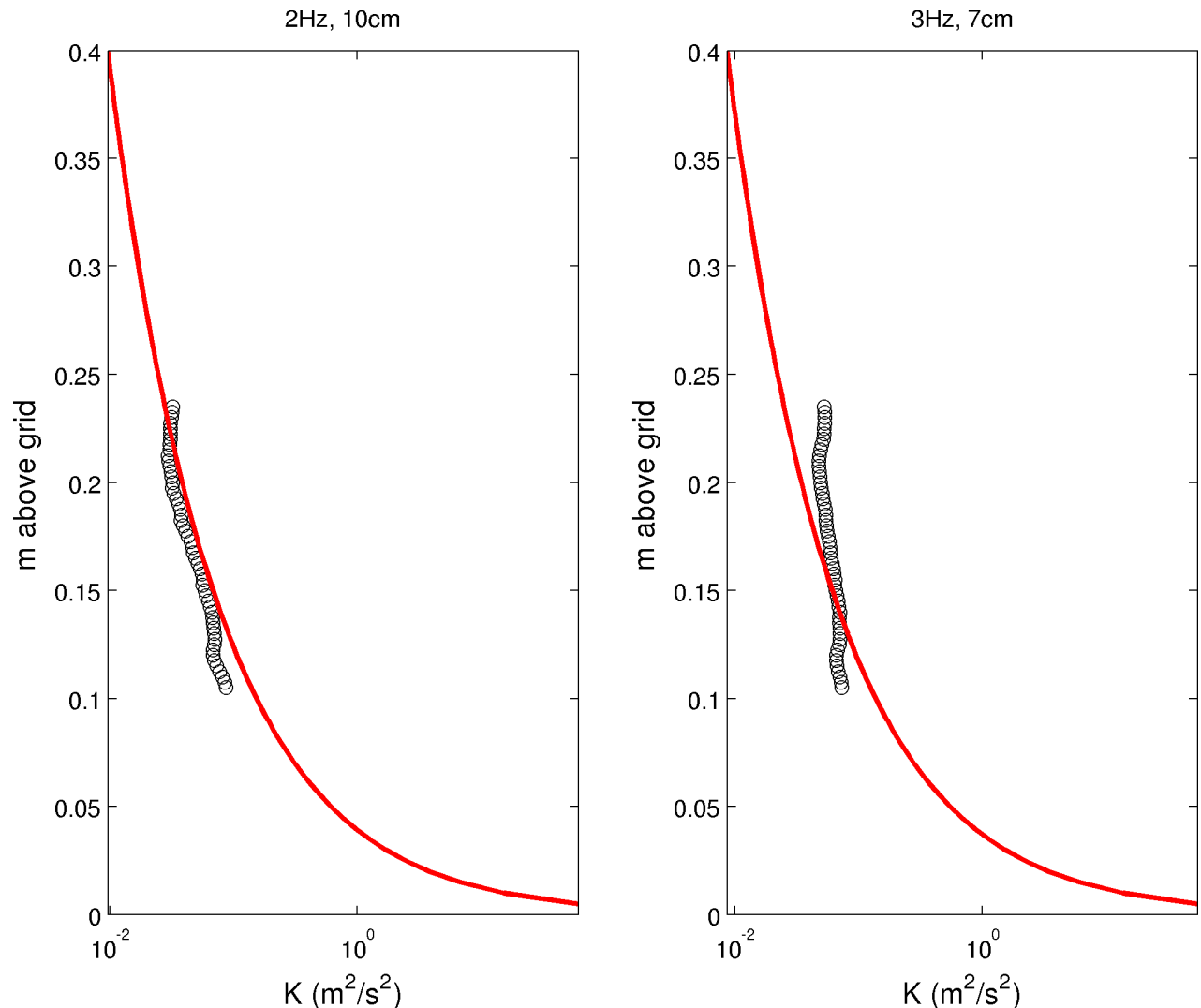

Figure 7. Turbulent kinetic energy profiles computed (symbols) from measured velocities using (10) and modelled (lines) using (15) and (16).

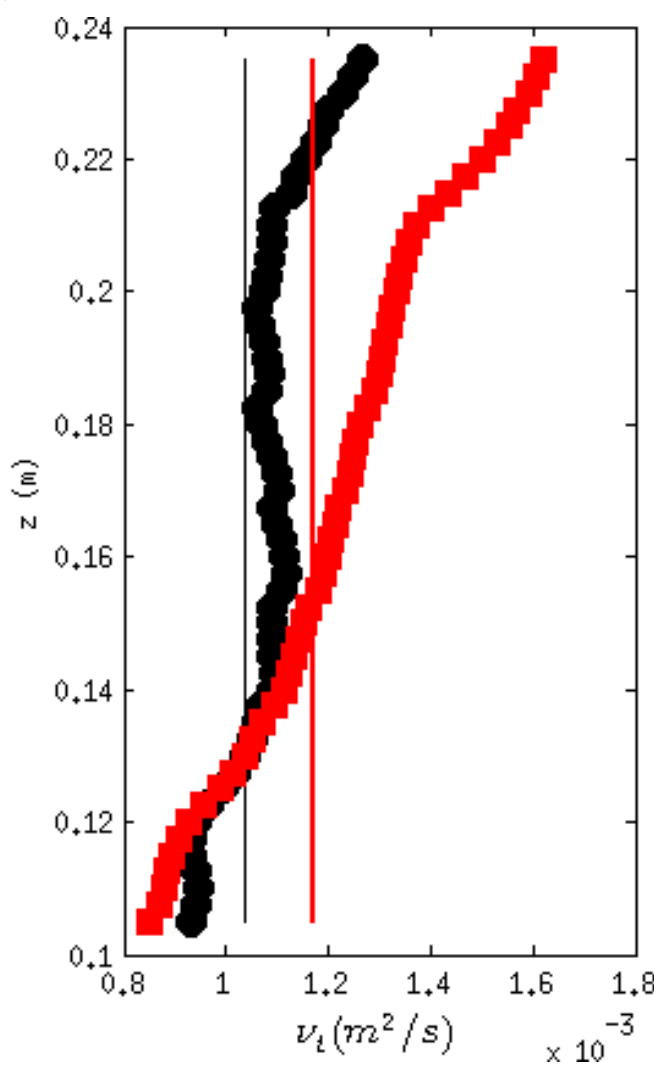

Figure 8. Measured (symbols) and modelled (lines) turbulent eddy viscosity for the $2 \mathrm{~Hz}$ (black) and $3 \mathrm{~Hz}$ (red) grid shaking. 
Following Michallet and Mory (2004) we express turbulent eddy viscosity as:

$$
v_{t}=\frac{C_{\mu} K^{2}}{\varepsilon}
$$

where turbulence dissipation rate uses a turbulent mixing length, $l$, and is given by:

$$
\varepsilon=C_{\mu}^{3 / 4} \frac{K^{3 / 2}}{l}
$$

Inserting (15) into (17) and for the integral length scale adopting $l=\lambda z$, as suggested by Turner (1968) and confirmed theoretically by Matsunaga et al. (1999) we obtain

$$
v_{t}=\sqrt{C_{\mu}} \sqrt{\frac{1}{2} \omega\left(S^{1.5} f z^{-1} \sqrt{M}\right)} \lambda z
$$

We use the standard stability parameter $C_{\mu}=0.09$ (Michallet and Mory, 2004) and adopt $\lambda=0.1$ following Turner (1968) and Medina et al. (2001). The model does not reproduce the measurements exactly (Figure 8) but does predicts the same order of magnitude and the same trend with respect to grid shaking (increased momentum diffusivity for the $3 \mathrm{~Hz}$ shaking). A possible reason for modelobservation mismatch is that $l=\lambda z$ is not an appropriate model or 0.1 not a suitable value for $\lambda$. Indeed, there is some debate as to the appropriate value for $\lambda$ (e.g. Matsunaga et al., 1999; Orlins and Gulliver, 2003), indeed whether there should be separate values for near-grid and far-grid regions (e.g. Guadayol et al., 2009). Future work will ascertain the correct length scale using spectral estimates of turbulent dissipation from the velocity data, using (Michallet and Mory, 2004):

$$
l=\frac{K^{3 / 2}}{\varepsilon}
$$
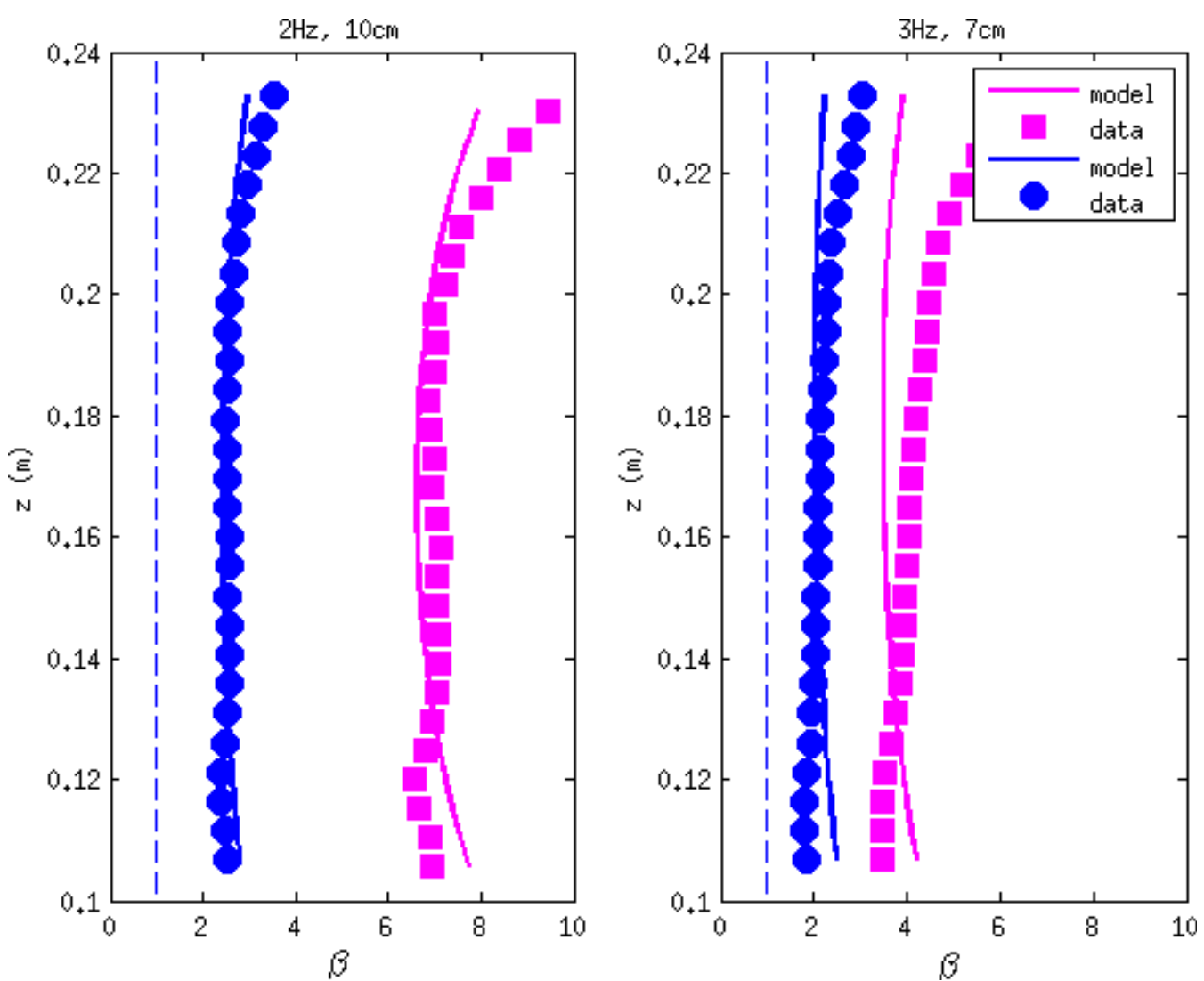

Figure 9. Schmidt numbers calculated for $2 \mathrm{~Hz}$ (lefft) and $3 \mathrm{~Hz}$ (right) flow regimes. Solid lines indicate the analytical expression for $\beta$ and symbols indicate values calculated from the data. 
Combining (14) and (19), the modelled Schmidt numbers (solid lines in Figure 9) show close agreement with Schmidt numbers calculated from measurements using (12), (17), and (18) (symbols in Figure 9). For both grid shaking conditions, and for both the fine and coarse sediment, $\beta>1$ which indicates that turbulent momentum is more diffusive than the sediment. The Schmidt numbers are relatively uniform with depth, and go with grid Reynolds number (5), with larger $\beta$ for the $2 \mathrm{~Hz}, 10 \mathrm{~cm}$ stroke $\left(\mathrm{Re}_{\text {grid }}=14705\right)$ than the $3 \mathrm{~Hz}, 7 \mathrm{~cm}$ stroke $\left(\operatorname{Re}_{\text {grid }}=10808\right)$.

\section{DISCUSSION}

Apparent grain-size dependency in calculated and modelled Schmidt numbers (Figure 9) is interesting. Nielsen and Teakle (2004) also observed greater $\beta$ for smaller grain diameters (inversely related to $D$ ), postulating that it could be explained by finer grains settling faster through turbulence than in still water, for which there is some experimental evidence. Alternatively, it is possible that the mixing length parameterization is inaccurate, and even that the mixing length for smaller grains is smaller than for the fluid.

However, given that the mean concentrations were higher for the finer sediment than for the coarser sediment (Figure 3), it is possible that $\beta$ goes with mean $C$ due to negative feedback processes, whereby as more sediment diffuses into the water it becomes harder for the sediment to suspend due to diminishing gradients, and the sediment diffusivity decreases (Lees, 1981; Amoudry et al., 2005). It seems less likely that sediment-induced buoyancy effects are the real cause of the apparent grain-size dependence. Suspended-sediment-induced density stratification would damp turbulence, thereby reducing the eddy viscosity and therefore one would expect $\beta$ to be positively related to $D$.

\section{CONCLUSIONS}

The apparent diffusivity of momentum is related to the apparent diffusivity of mass through the turbulent Schmidt number. Bycreating a zero-mean flow and near-isotropic turbulence using an oscillating grid turbulence tank, we can create stationary suspensions of sediment under pure gradient diffusion. Detailed measurements were made of vertical profiles of suspension sediment concentration, grain size and the 3 components of velocity. By varying the grid shaking and grain size characteristics, we calculated sediment and mometum diffusivities directly under a range of representative conditions. Initial results suggest that momentum diffusivity is greater than sediment diffusivity, and that the ratio of the two scales with grid Reynolds number. Ongoing work will ascertain whether an apparent grain size dependence could instead be explained by two-way feedbacks between sediment and turbulence.

\section{ACKNOWLEDGMENTS}

The work was funded by a (UK) Natural Environment Research Council grant (NE/G007543/1) awarded to D.C.

\section{REFERENCES}

Absi, R. 2010. Concentration profiles for fine and coarse sediments suspended by waves over ripples: an analytical study with the 1-DV gradient diffusion model, Advances in Water Resources, 33, 411-418.

Amoudry, L., T.-J. Hsu, P.L.-F. Liu. 2005. Schmidt number and near-bed boundary condition effects on a two-phase dilute sediment transport model, Journal of Geophysical Research, 110, C09003.

Betteridge, K.F.E., P.D. Thorne, and R.D. Cooke. 2008. Calibrating multi-frequency acoustic backscatter systems for studying near-bed suspended sediment transport processes, Continental Shelf Research, 28, 227-235.

Celik, I., and W. Rodi. 1988. Modeling suspended sediment transport in non equilibrium situations, Journal of Hydraulic Engineering, 114, 101157-101191.

Conley, D.C., and R. Beach. 2003. Cross-shore partitioning in the nearshore during a storm event, Journal of Geophysical Research, 108, C3, 3065.

Conley, D.C., S. Flachetti, I.P. Lohmann, and M. Brocchini. 2008. The effects of flow stratification by non-cohesive sediment on transport in high-energy wave-driven flows, Journal of Fluid Mechanics, 610, 43-67.

Davies, A.G. 1995. Effects of unsteadiness on the suspended sediment flux in co-linear wave-current flow, Continental Shelf Research, 15, 949-979.

Davies, A.G., and P.D. Thorne. 2005. Modelling and measurement of sediment transport by waves in the vortex ripple regime, Journal of Geophysical Research, 110, C05017.

deSilva, I.P.D., and H.J.S. Fernando. 1992. Oscillating grids as a source of nearly isotropic 
turbulence, Physics of Fluids, 6, 2455-2464.

Dohmen-Janssen, C.M., W.N. Hassan, J.S. Ribberink. 2001. Mobile-bed effects in oscillatory sheet flow, Journal of Geophysical Research, 106, 27103-27115.

Ferguson, R.I., and M. Church. 2004. A simple universal equation for grain settling velocity, Journal of Sedimentary Research, 74, 933-937.

Fredsoe, J., O.H. Andersen, and S. Silberg. 1985. Distribution of suspended sediment in large waves, Journal of Waterway, Port, Coastal and Ocean Engineering, 11, 1041-1059.

Gaunaurd, G.C., and H. Uberall. 1983. RST analysis of monostatic and bistatic acoustic echoes from an elastic sphere, The Journal of the Acoustic Society of America, 73, 1-12.

Graf, W.H., and M. Cellino. 2002. Suspension flows in open channels: an experimental study, Journal of Hydraulic Research, 435-437.

Gratiot, N., H. Michallet, M. Mory. 2005. On the determination of the settling flux of cohesive sediments in a turbulent fluid, Journal of Geophysical Research, 110, C06004.

Guadayol, O., F. Peters, J.E. Stiansen, C. Marrase, and A. Lohrmann. 2009. Evaluation of oscillating grids and orbital shakers as means to generate isotropic and homogeneous small-scale turbulence in laboratory enclosures commonly used in plankton studies, Limnology and Oceanography: Methods, 7, 287-303.

Henderson, S.M., J.S. Allen, and P.A. Newberger. 2004. Nearshore sandbar migration predicted by an eddy-diffusive boundary layer model, Journal of Geophysical Research, 109, C06024.

Holmedal, L.E., D. Myrhaug, and K.J. Eidsvik. 2004. Sediment resuspension under sheet flow conditions beneath random waves plus current. Continental Shelf Research, 24, 2065-2091.

Hopfinger, E.J., and J.A. Toly. 1976. Spatially decaying turbulence and its relation to mixing across density interfaces, Journal of Fluid Mechanics, 78, 155-175.

Lees, B.J. 1981. Relationship between eddy viscosity of seawater and eddy diffusivity of suspended particles, Geo-Marine Letters, 1, 249-254.

Li, Z., and A.G. Davies. 2001. Turbulence closure modelling of sediment transport beneath large waves. Continental Shelf Research, 21, 243-262.

Matsunaga, N., Y. Sugihara, T. Komatsu, and A. Masuda. 1999. Quantitative properties of oscillating-grid turbulence in a homogeneous fluid, Fluid Dynamics Research, 25, 147-165.

Medina, P., M.A. Sanchez, and J.M. Redondo. 2001. Gris stirred turbulence: Applications to the iniation of sediment motion and lift-off studies, Physics and Chemistry of the Earth (B), 26, 299304.

Michallet, H., and M. Mory. 2004. Modelling of sediment suspensions in oscillating grid turbulence, Fluid Dynamics Research, 35, 87-106.

Nielsen, P., and I.A.L. Teakle. 2004. Turbulent diffusion of momentum and suspended particles: a finite-mixing-length-theory, Physics of Fluids, 16, 2342-2348.

Orlins, J.J., and J.S. Gulliver. 2003. Turbulence quantification and sediment resuspension in an oscillating grid chamber, Experiments in Fluids, 34, 662-677.

Redondo, J.M., X. Durrieu de Madron, P. Medina, M.A. Sanchex, and E. Schaaff. 2001. Comparison of sediment resuspension measurements in sheared and zero-mean turbulent flows, Continental Shelf Research, 21, 2095-2103.

Ruessink, B.G., T.J.J. Van den Berg, and L.C. Van Rijn. 2009. Modeling sediment transport beneath skewed asymmetric waves above a plane bed, Journal of Geophysical Research, 114, C11021.

Thorne, P.D., A.G. Davies, and P.S. Bell. 2009. Observations and analysis of sediment diffusivity profiles over sandy rippled beds under waves, Journal of Geophysical Research, 114, C02023.

Thorne, P.D., and D.M. Hanes. 2002. A review of acoustic measurement of small-scale sediment processes, Continental Shelf Research, 22, 603-632.

Turner, J.S. 1968. The influence of molecular diffusivity on turbulent entrainment across a density interface, Journal of Fluid Mechanics, 33, 639-656.

van Rijn, L.C. 1984. Sediment transport, part II: Suspended load transport, Journal of Hydraulic Engineering, 110, 1613-1641. 